\title{
Orbital lymphatic-venous malformation with concomitant spontaneous orbital arteriovenous fistula: case report
}

\author{
Bunyada Putthirangsiwong, MD, 1 Dinesh Selva, FRACS, FRANZCO, ${ }^{2}$ \\ Weerawan Chokthaweesak, MD, ${ }^{1}$ Ekachat Chanthanaphak, MD, ${ }^{3}$ and \\ Sirintara Singhara Na Ayudhaya, MD
}

\begin{abstract}
Departments of ${ }^{1}$ Ophthalmology and ${ }^{3}$ Radiology, Ramathibodi Hospital, Mahidol University, Bangkok, Thailand; and ${ }^{2}$ Department of Ophthalmology and Visual Sciences, University of Adelaide and South Australian Institute of Ophthalmology, Adelaide, Australia
\end{abstract}

\begin{abstract}
The authors describe the case of an 11-year-old boy with no history of prior trauma, who experienced acute proptosis and visual loss while showering. Diagnostic carotid artery angiography revealed an orbital lymphatic-venous malformation (LVM) concomitant with an intraorbital arteriovenous fistula. The patient was treated with transvenous coil and $\mathrm{N}$-butyl cyanoacrylate glue embolization, combined with direct percutaneous glue injection into the LVM followed by excision. There was good clinical and radiological response without recurrence at the 2-year follow-up. Diagnostic carotid angiography should be considered in cases of orbital LVM, as a concomitant arteriovenous fistula might be present in rare cases.
\end{abstract}

https://thejns.org/doi/abs/10.3171/2017.8.PEDS17379

KEY WORDS orbital lymphatic/lymphatic-venous malformations; intraorbital arteriovenous fistula; vascular malformation; embolization; vascular disorders

$\mathrm{O}$ RBITAL lymphatic-venous malformations (LVMs), so-called lymphangiomas, are currently classified based on the International Society for the Study of Vascular Anomalies classification as slow-flow vascular malformations and represent $1 \%-3 \%$ of all orbital masses. ${ }^{8,13}$ The majority are mixed LVMs and have a variable venous component with a variable systemic venous outflow. ${ }^{8,9}$ Because of their infiltrative nature, location, and relationships to adjacent vital structures of the orbit, management can be challenging.

An intraorbital arteriovenous fistula (AVF) is a very rare, high-flow vascular malformation characterized by a direct connection between the ophthalmic artery and one of the draining ophthalmic veins, such as the superior ophthalmic vein (SOV), inferior ophthalmic vein, or facial vein without an intervening capillary nidus. ${ }^{6}$ The etiological mechanism of an intraorbital AVF may be traumatic or spontaneous. ${ }^{15}$ Because of the rarity of case reports, the standard treatment of an intraorbital AVF has not been established. Herein, we describe a case of an orbital LVM with a coexisting spontaneous intraorbital AVF.

\section{Case Report}

An 11-year-old boy presented with acute, severe proptosis and sudden visual loss in his right eye that occurred while showering. The initial diagnosis was orbital compartment syndrome, and the patient underwent lateral canthotomy, cantholysis, and emergency CT imaging of the orbit at a local rural hospital. He was given intravenous methylprednisolone $500 \mathrm{mg}$ once daily, $0.5 \%$ timolol eye drop every 12 hours, $0.02 \%$ brimonidine-P eye drop every 12 hours, oral acetazolamide $250 \mathrm{mg}$ every 8 hours, and $50 \%$ glycerin $50 \mathrm{ml}$ every 8 hours. CT scanning showed a large right intraconal orbital lesion with an internal bloodfluid level and intralesional enhancement in the superior orbit. The next day, the patient, who had a presumed diagnosis of an LVM with acute intralesional hemorrhage, was referred to our institution. At the age of 3 years, the patient began exhibiting intermittent proptosis of his right eye while performing Valsalva maneuver activities. There was no history of trauma.

Examination revealed no light perception on the right,

ABBREVIATIONS AVF = arteriovenous fistula; $L \mathrm{LM}=$ lymphatic-venous malformation; SOV $=$ superior ophthalmic vein .

SUBMITTED July 7, 2017. ACCEPTED August 16, 2017.

INCLUDE WHEN CITING Published online November 24, 2017; DOI: 10.3171/2017.8.PEDS17379. 

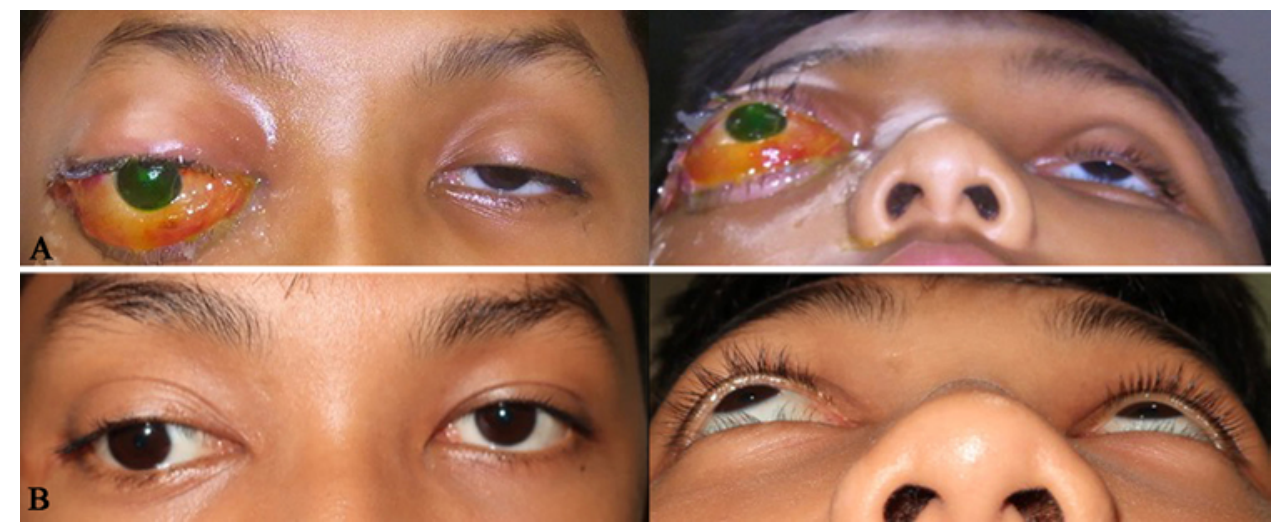

FIG. 1. A: Photographs obtained before treatment, showing marked proptosis and chemosis of the patient's right eye. B: Photographs obtained 3 months after treatment, showing clinical improvement. Figure is available in color online only.

with a fixed, dilated pupil and a positive reverse relative afferent pupillary defect. The patient exhibited marked proptosis and chemosis (Fig. 1A). The intraocular pressure was $25 \mathrm{~mm} \mathrm{Hg}$. The fundal view was limited due to dense corneal punctate epithelial erosions and corneal abrasion. Extraocular movements were limited in all directions. Findings from the left-eye ophthalmic examination were normal.

The orbit was isointense on T1-weighted MRI, and the lesion appeared mixed hypo-/hyperintense on T2-weighted MRI, with internal hemorrhage seen in the gradient echo sequence involving both intraconal and extraconal spaces of the right orbit that extended posteriorly into the superior orbital fissure. There was a peripheral thin rim and septal enhancement (Fig. 2A). An LVM with acute intralesional bleeding was suspected. The patient underwent diagnostic angiography after induction of general anesthesia, which revealed a contrast-filled lesion in the right orbit that was fed from the orbital branch of the right ophthalmic artery and drained into the right SOV and right cavernous sinus, consistent with an AVF (Fig. 3 ). Direct puncture using a 20-gauge catheter (Jelco) under fluoroscopic guidance was performed in the medial 2 cysts of the LVM in preparation for bleomycin injection. Then, Hexabrix $(320 \mathrm{mg} / \mathrm{ml})$ contrast was injected to localize the position of the Jelco tip and demonstrated contrast drainage to the right SOV and right cavernous sinus. The diagnosis of an orbital LVM with an intraorbital AVF was made. Thus, the catheter was withdrawn, and the strategy was changed. Treatment was initiated via a transvenous approach to obliterate the fistula first before direct puncture with bleomycin injection. A 2.3-Fr Prowler Plus microcatheter (Codman Neuro) was navigated into the right $\mathrm{SOV}$, and microcoil embolization was done using 1 Tornado embolization coil $(3 \mathrm{~mm} \times 2 \mathrm{~cm}$, Cook Medical) and 14 Nester embolization coils $(3 \mathrm{~mm}$ $\times 14 \mathrm{~cm}$, Cook Medical). Postembolization angiography demonstrated $90 \%$ flow reduction in the intraorbital AVF.

Direct puncture with contrast injection under fluoroscopic guidance was done again into the same medial cyst, which still revealed slow-flow venous drainage to the right SOV and right cavernous sinus. Furthermore, the cavity was freely aspirated, which resulted in obtaining $50 \mathrm{ml}$ of blood, indicating a residual fistula and incomplete outflow occlusion. Therefore, plans for sclerosing therapy of the LVM were abandoned.

Clinical improvement from thrombotic effects at the interventional site was expected. However, 1 week later, the patient had no clinical improvement. He underwent cerebral angiography, which showed minimal residual slow flow in the AVF with venous drainage into the right SOV and right cavernous sinus. After a multidisciplinary meeting, we decided to proceed with surgical removal of the LVM after glue embolization instead of intralesional bleomycin injection due to the inability to close the AVF completely. Transfemoral transvenous glue embolization via the right SOV was combined with percutaneous intralesional glue injection at the superomedial and inferomedial areas of the LVM using 33\% mixtures of $N$-butyl cyanoacrylate with lipiodol. Volumes of $1.5 \mathrm{ml}$ (in the AVF), $2.5 \mathrm{ml}$ (in the superomedial portion of the LVM), and 2.0 $\mathrm{ml}$ (in the inferomedial portion of the LVM) were injected in an attempt to close the systemic connection and enable excision. Excision of the superomedial and inferomedial components was performed 5 days later via a lateral orbitotomy. Histopathological examination revealed multiple dilated sacs lined with a single layer of endothelium of the LVM with organized thrombus and glue. At the 1-week follow-up, the patient's vision was unchanged, but his proptosis and chemosis were dramatically diminished.

At the 3-month follow-up, the patient exhibited $3 \mathrm{~mm}$ of proptosis with minimal supraduction and abduction deficit. There was a significant decrease in the size of the LVM on repeated MRI of the orbit (Figs. 1B and 2B). The patient has been stable with no recurrence of the LVM or intraorbital AVF during a 2-year follow-up period.

\section{Discussion}

Orbital LVMs are rare, benign, congenital vascular malformations. These lesions may be seen at birth but are generally not clinically apparent until infancy or childhood. They may present after an upper respiratory tract infection, trauma, or with acute intralesional bleeding. The most common presentations are proptosis, ptosis, swelling of periorbital soft tissue, and restriction of eye movement. ${ }^{8}$ 

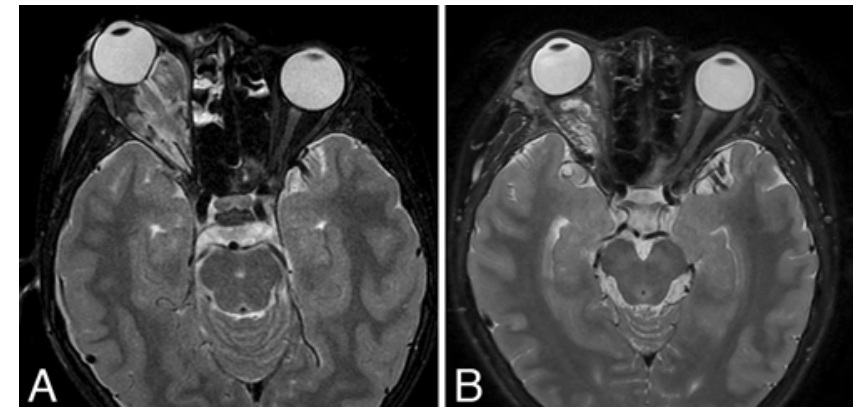

FIG. 2. Axial T2-weighted MR images. A: Image obtained before treatment, demonstrating an intraconal mixed hypo-/hyperintense lesion with a peripheral thin rim and septal enhancement of the right orbit. B: At the 3-month follow-up, a markedly decreased size of the LVM is revealed.

There are various systems of classification of orbital LVMs. These lesions are categorized as no-flow to slowflow lesions based on the Orbital Society recommendations. ${ }^{5}$ According to the International Society for the Study of Vascular Anomalies classification, they are classified as slow-flow malformations. ${ }^{13}$ In terms of location, orbital LVMs are further grouped as superficial, deep, combined, and complex. ${ }^{11}$ Rootman et al. ${ }^{9}$ considered the hemodynamic effect of size and type of inflow and outflow channels important. Therefore, orbital lesions have been categorized as pure lymphatic malformations and combined LVMs. LVMs are relatively more common than pure lymphatic malformations and have a variable venous component with a variable range of connection to the systemic venous circulation. Combined lymphatic-dominant lymphatic malformations and pure lymphatic malformations demonstrate little or no evidence of systemic venous outflow, while combined venous-dominant LVMs can have a large venous outflow to the systemic circulation. ${ }^{9}$ Histologically, orbital LVMs have multiple dilated sacs and lobules lined with a single layer of endothelium, containing a proteinaceous or serosanguinous fluid with connective tissue stroma, smooth muscle bundles, dense small lymphocytes or follicles, and stromal hemorrhage. ${ }^{8}$

Regarding the correlation between orbital LVMs and other vascular malformations, there is a significant association between diffuse orbital LVMs and noncontiguous intracranial vascular anomalies, including developmental venous anomalies, dural arteriovenous malformations, occlusion or absence of dural sinuses and jugular veins, cerebral cavernous malformations, and pial arteriovenous malformations. ${ }^{1}$ Coll et al. reported a case of concomitant medial extraconal orbital lymphangioma and ipsilateral arteriovenous malformation of the orbit and middle cranial fossa. ${ }^{2}$ Coumou et al. reported a case of a carotid-cavernous dural AVF concomitant with a venous/lymphatic malformation. ${ }^{3}$ To the best of our knowledge, we describe the first case of an orbital LVM with concomitant spontaneous orbital AVF.

Intraorbital AVFs are a challenge to diagnose and manage. The clinical manifestations, including eye redness, proptosis, visual loss, diplopia, orbital pain, and bruits, may mimic those of carotid-cavernous sinus fistulas. ${ }^{7}$ The etiology of a traumatic intraorbital AVF is a penetrating

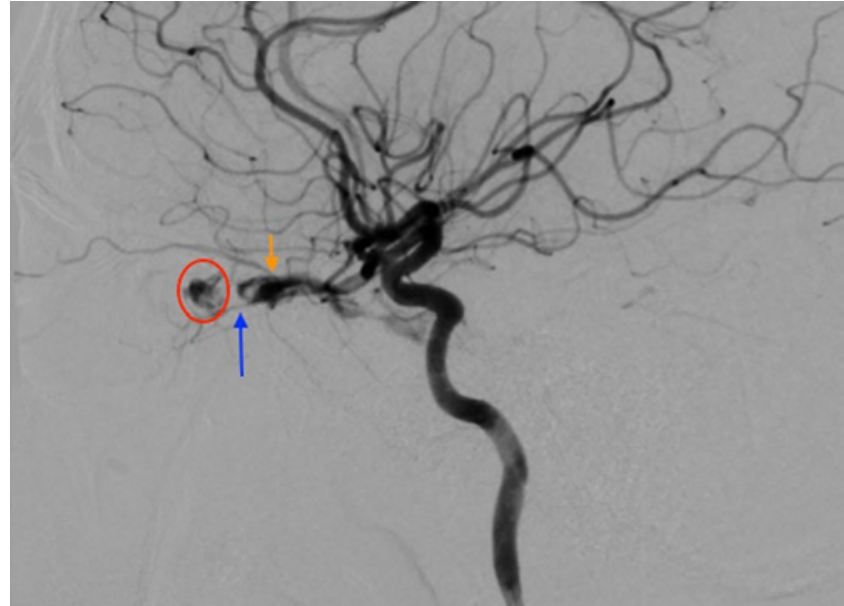

FIG. 3. Diagnostic cerebral angiogram revealing a contrast-filled lesion in the right orbit representing an AVF (red circle), which was fed by the right ophthalmic artery (blue arrow) and drained into the right SOV (orange arrow). Figure is available in color online only.

injury causing perforation of both the artery and vein where they cross each other, whereas the mechanism of a spontaneous orbital AVF is not clear. Theories include a preexisting ophthalmic artery aneurysm that ruptures into an adjacent vein or gradual mechanical erosion of an artery from hypertension, atherosclerosis, or other vascular diseases. ${ }^{15}$ In our case, we postulate that the mechanism of this spontaneous intraorbital AVF could be from the local mechanical erosion of the ophthalmic artery caused by the multiple lobulated masses of the orbital LVM.

The management of both orbital LVMs and orbital AVFs is challenging. Orbital LVMs are irregular, infiltrative lesions involving adjacent vital structures of the orbit. They are rich with vascular beds, which can lead to uncontrolled bleeding and may also have no accessible vascular channels for endovascular approaches. ${ }^{12}$ Conservative treatment is recommended for lesions that cause no visual deficits or cosmetic issues. The nonsurgical strategies previously reported for orbital LVMs are sclerosing therapy including ethanol, OK-432, sodium tetradecyl sulfate (Sotradecol, Mylan), morrhuate sodium, doxycycline, bleomycin, and pinyangmycin. ${ }^{8,11,12} \mathrm{~A}$ carbon dioxide laser, beta irradiation, and adjunctive systemic corticosteroids are also options for treatment. ${ }^{8}$ Recently, the use of oral sildenafil for the treatment of microcystic lesions has been reported. ${ }^{8}$ Surgical management can be combined with preoperative intralesional injection of sclerosing agents (e.g., morrhuate sodium) or liquid polymers (e.g., $N$-butyl cyanoacrylate glue or fibrin glue) to aid in dissection and improve hemostasis. ${ }^{8,11}$ In the case of an orbital AVF, small low-flow lesions may have spontaneous remission or resolution after diagnostic angiography. ${ }^{15}$ Conservative treatment is favored in those with no significant symptoms. In cases with progressive orbital congestive symptoms and visual deficit, treatment with endovascular embolization (via transvenous, transarterial, or direct surgical approaches) ${ }^{7}$ is an option. Alternative treatment strategies include embolization followed by 
resection ${ }^{10}$ or surgery alone. ${ }^{4,14}$ The standard treatment is not well established due to the rarity of these lesions, but transvenous embolization with coils, oxidized cellulose (Surgicel, Ethicon), or Onyx ${ }^{7}$ has been most frequently reported with satisfactory outcomes. In our patient, the angiogram demonstrated both the orbital LVM with venous outflow drainage to systemic circulation and an orbital AVF; therefore, a combination of treatments was used. We performed a transfemoral transvenous approach via the right SOV for coil embolization of the fistula and venous outflow drainage of the LVM to close the systemic connection and inject the bleomycin. However, the fistula remained partially patent, and the outflow occlusion was incomplete. Hence, further transfemoral transvenous embolization with cyanoacrylate glue instead of bleomycin was used to close the residual fistula and venous outflow, posteriorly combined with percutaneous intralesional glue injection into the LVM anteriorly. Resection of the LVM was performed in the same admission to avoid a foreign body reaction caused by cyanoacrylate glue.

\section{Conclusions}

An intraorbital AVF can be found to coexist with lowflow orbital LVMs. Diagnostic carotid angiography should be considered in cases of orbital LVMs to exclude abnormal high-flow shunting. In our case, preoperative CT scanning and MRI did not reveal this high-flow abnormality. Management by a multidisciplinary team involving oculoplastic surgeons and interventional neuroradiologists is essential for the optimal management of these lesions.

\section{References}

1. Bisdorff A, Mulliken JB, Carrico J, Robertson RL, Burrows PE: Intracranial vascular anomalies in patients with periorbital lymphatic and lymphaticovenous malformations. AJNR Am J Neuroradiol 28:335-341, 2007

2. Coll GE, Goldberg RA, Krauss H, Bateman BJ: Concomitant lymphangioma and arteriovenous malformation of the orbit. Am J Ophthalmol 112:200-205, 1991

3. Coumou AD, van den Berg R, Bot JC, Beetsma DB, Saeed P: Direct orbital puncture of the cavernous sinus for the treatment of a carotid-cavernous dural AV fistula with a concomitant venous/lymphatic malformation. Orbit 33:68-71, 2014

4. Hamada J, Morioka M, Kai Y, Sakurama T, Kuratsu J: Spontaneous arteriovenous fistula of the orbit: case report. Surg Neurol 65:55-57, 2006

5. Harris GJ: Orbital vascular malformations: a consensus statement on terminology and its clinical implications. Am J Ophthalmol 127:453-455, 1999

6. Kim AW, Kosmorsky GS: Arteriovenous communication in the orbit. J Neuroophthalmol 20:17-19, 2000
7. Lv X, Li W, Liu A, Lv M, Jiang C: Endovascular treatment evolution for pure intraorbital arteriovenous fistula: three case reports and literature review. Neuroradiol J 30:151159,2017

8. Nassiri N, Rootman J, Rootman DB, Goldberg RA: Orbital lymphaticovenous malformations: current and future treatments. Surv Ophthalmol 60:383-405, 2015

9. Rootman J, Heran MK, Graeb DA: Vascular malformations of the orbit: classification and the role of imaging in diagnosis and treatment strategies. Ophthal Plast Reconstr Surg 30:91-104, 2014

10. Rootman J, Kao SC, Graeb DA: Multidisciplinary approaches to complicated vascular lesions of the orbit. Ophthalmology 99:1440-1446, 1992

11. Saha K, Leatherbarrow B: Orbital lymphangiomas: a review of management strategies. Curr Opin Ophthalmol 23:433438,2012

12. Stacey AW, Gemmete JJ, Kahana A: Management of orbital and periocular vascular anomalies. Ophthal Plast Reconstr Surg 31:427-436, 2015

13. Wassef M, Blei F, Adams D, Alomari A, Baselga E, Berenstein A, et al: Vascular anomalies classification: recommendations from the International Society for the Study of Vascular Anomalies. Pediatrics 136:e203-e214, 2015

14. Wigton EH, Wells JR, Harrigan MR, Long JA, Vicinanzo MG: Diagnosis and management of an intraorbital vascular fistula. Ophthal Plast Reconstr Surg 28:e39-e41, 2012

15. Yazici B, Yazici Z, Erdogan C, Rootman J: Intraorbital arteriovenous fistula secondary to penetrating injury. Ophthal Plast Reconstr Surg 23:275-278, 2007

\section{Disclosures}

The authors report no conflict of interest concerning the materials or methods used in this study or the findings specified in this paper.

\section{Author Contributions}

Conception and design: Putthirangsiwong, Selva. Acquisition of data: Putthirangsiwong. Analysis and interpretation of data: Putthirangsiwong. Drafting the article: Putthirangsiwong. Critically revising the article: Chanthanaphak, Putthirangsiwong, Selva, Chokthaweesak. Reviewed submitted version of manuscript: all authors. Approved the final version of the manuscript on behalf of all authors: Chanthanaphak. Administrative/technical/material support: Chanthanaphak, Chokthaweesak, Singhara Na Ayudhaya. Study supervision: Chanthanaphak, Selva, Chokthaweesak, Singhara Na Ayudhaya.

\section{Correspondence}

Ekachat Chanthanaphak, Department of Radiology, Faculty of Medicine, Ramathibodi Hospital, Mahidol University, 270 Rama VI Rd., Ratchathewi, Bangkok 10400, Thailand. email: potter_ ra@hotmail.com. 